\title{
Filigrane
}

Écoutes psychothérapiques

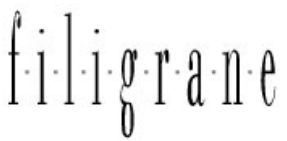

\section{La résistance actuelle}

\section{Claudette Lafond}

Volume 17, numéro 2, automne 2008

L’avenir du clinicien II

URI : https://id.erudit.org/iderudit/019415ar

DOI : https://doi.org/10.7202/019415ar

Aller au sommaire du numéro

\section{Éditeur(s)}

Revue Santé mentale au Québec

ISSN

1192-1412 (imprimé)

1911-4656 (numérique)

Découvrir la revue

\section{Citer cet article}

Lafond, C. (2008). La résistance actuelle. Filigrane, 17(2), 6-18. https://doi.org/10.7202/019415ar

\section{Résumé de l'article}

Cet article est une invitation à réfléchir sur les manifestations de la résistance et du dogmatisme à la fois chez les cliniciens et dans les politiques de santé mentale. Le propos met en évidence les répercussions de ces manifestations qui influencent le clinicien d'orientation psychodynamique. Il énonce les collusions possibles avec l'idéologie ambiante caractérisée par les impératifs économiques et les impératifs d'intégration sociale. L'auteure souligne l'hétérogénéité entre l'approche analytique et celles qui se veulent scientifiques d'inspiration positiviste, tentant ainsi de montrer l'incompatibilité des finalités poursuivies. À partir de ces constats, l'avenir du clinicien d'orientation analytique se concevrait par l'affirmation de son identité en phase avec l'héritage subversif de la psychanalyse et par l'obligation de rendre compte de son travail dans le social.
Ce document est protégé par la loi sur le droit d'auteur. L'utilisation des services d'Érudit (y compris la reproduction) est assujettie à sa politique d'utilisation que vous pouvez consulter en ligne.

https://apropos.erudit.org/fr/usagers/politique-dutilisation/ 


\title{
La résistance actuelle
}

\author{
claudette lafond
}

Cet article est une invitation à réfléchir sur les manifestations de la résistance et du dogmatisme à la fois chez les cliniciens et dans les politiques de santé mentale. Le propos met en évidence les répercussions de ces manifestations qui influencent le clinicien d'orientation psychodynamique. Il énonce les collusions possibles avec l'idéologie ambiante caractérisée par les impératifs économiques et les impératifs d'intégration sociale. L'auteure souligne l'hétérogénéité entre l'approche analytique et celles qui se veulent scientifiques d'inspiration positiviste, tentant ainsi de montrer l'incompatibilité des finalités poursuivies. À partir de ces constats, l'avenir du clinicien d'orientation analytique se concevrait par l'affirmation de son identité en phase avec l'héritage subversif de la psychanalyse et par l'obligation de rendre compte de son travail dans le social.

\section{État de la question}

a plupart des doléances des cliniciens d'approche psychodynamique, ciblent l'environnement institutionnel des services de santé, sous l'emprise de restrictions budgétaires et de thérapies comportementales. Ajoutons à cela que les départements de psychologie des universités sont peu enclins à former des thérapeutes d'orientation analytique. C'est au nom d'une certaine rigueur scientifique que l'on justifie les choix théoriques sans questionner la représentation du patient formalisée derrière le modèle véhiculé par cette supposée rigueur scientifique. Il est probable que la science, à partir de laquelle on aspirait à maîtriser, à dominer la nature, et, éventuellement, l'humain, ambitionne d'enrayer tout dysfonctionnement à partir de ce qu'on décrète être le bonheur de chacun. Bonheur qui se conjugue avec la prospérité économique.

Freud avait jadis affirmé que la chimie et la biologie apporteraient, un jour, des solutions aux problèmes que la psychanalyse essaie d'expliquer ou de résoudre. Il nous invitait à psychanalyser le plus possible, c'est-à-dire tant que l'inconscient demeure accessible (Granoff, 2001, 157). Or, le savoir tributaire de l'écoute de l'inconscient peut devenir inaccessible dans la mesure où une fin de non-recevoir est brandie par un dogme de scientificité syntone avec des prérogatives économiques. Deux effets résultent de cette configuration. Le premier est de fortifier la résistance à l'écoute de l'inconscient, le deuxième, de manipuler le désir de l'être souffrant, ou, à tout le moins, de l'orienter et de le traduire en phase avec les besoins socio-économiques.

«Le discours économique, d'un côté, fait l'apologie du désir comme source énergétique de la richesse et principe du calcul de la valeur; de l'autre, il fait de ce même calcul le mode de régulation du désir en le rendant compatible avec le nouvel ordre des choses. » (Laval, 2007, 189) 
Le nouvel ordre des choses au service de la résistance participe de l'alliage de l'économie et de la science. Nous entrons dans l'idéologie, celle qui détermine le comportement de l'individu et de la société. Freud (1925), dans son article sur les résistances à la psychanalyse, dénonce les deux formes d'épistémè qui, depuis les débuts de la psychanalyse, ont constitué des résistances majeures : la pensée médicale avec son souci «matérialiste», «mécaniciste» et la pensée philosophique, avec sa psychologie de la conscience et son refus de l'inconscient. La philosophie s'est enfermée dans l'illusion d'un sujet unique, indivisible, d'un moi maître chez lui, et c'est précisément ce que Freud est venu déconstruire. Cette déconstruction heurte le narcissisme du rationaliste dans son travail dit scientifique. Et avec l' «évolution », le maître chez lui du rationaliste est devenu le maître chez l'autre de l'idéologie. Idéologie qui infiltre les universités, les hôpitaux et les CLSC. Or, nous savons que la résistance à la psychanalyse est connaturelle à la pratique de la psychanalyse. Nous résistons tous à l'inconscient, précisément pour sauvegarder l'intégrité du moi. Aussi, nous ne nous étonnerons pas de constater que l'environnement culturel n'est que l'effet extériorisé d'un mouvement intrapsychique. L'air du temps, pour ne pas dire de tout temps, nous rend à contretemps. Ce constat ne nous autorise cependant pas à mettre en veilleuse la possibilité de nous avouer que nous ne sommes pas uniquement à contretemps, mais également de notre temps et susceptibles d'être porteurs des bruits du monde anti-analytique en collusion avec nos propres résistances à l'écoute de l'inconscient qui, elles, sont hors temps.

\section{Identité en péril}

Dans cette perspective, mon propos se veut une invitation à réfléchir sur la question suivante: quelle est la constitution du désir anti-analytique dans l'environnement intellectuel et curatif. Question fondée sur l'a priori que l'opposant ne se situe pas exclusivement à l'extérieur. Il ne s'agit pas uniquement d'en prendre conscience, mais de reconnaître les effets de l'inconscient à l'œuvre. Cette reconnaissance survient sur fond de méconnaissance. Mon propos vise la connivence entre l'externe et l'interne. Une autre manière de laisser entendre que l'avenir du clinicien relève d'abord de sa prérogative. Je pense précisément au maintien de son identité et à ses conséquences, non seulement sur son travail thérapeutique, mais aussi sur son discours ou son silence dans le socius. À l'opposé, renoncer à son identité, c'est s'aliéner au discours de l'autre. Tel serait la cause sous-jacente de la morosité du clinicien dubitatif par rapport à son avenir qui, pour certains, s'exprime dans une tonalité dépressive avec plus ou moins de gravité.

[...] la résistance, que Freud appelle très tôt «la force de répulsion », impose d'emblée une étude subtile de ses rapports avec l'inconscient et le refoulé. [....], la résistance est soutenue, après 1920, par la jouissance masochiste et par l'emprise déliante des pulsions de mort. » (Chabert, 2002, 9) 
Il faut donc, pour le thérapeute, prendre en compte «cette force de répulsion » qui infiltre à la fois ses élaborations et sa collaboration à l'humeur ambiante. Par ailleurs, on ne peut se limiter à la notion d'identité, parce que ce qui importe, c'est de savoir ce qu'il y a au cœur de cette identité qui l'amènerait à s'aliéner au discours dominant, ce que certains jugeront comme une traitrise, la perte de son âme, alors que d'autres y verront une nécessité afin de pouvoir vivre de son métier. Une autre avenue demeure possible: la fuite. Une retraite qui assure une distance de la cité, afin de se retrouver entre sympathisants dans nos associations, nos colloques, à l'abri de nos attaquants, là où notre intégrité est sauvegardée. Le bureau privé peut également servir de refuge.

Le consentement à l'aliénation suppose une certaine séduction ou, à tout le moins, une fuite du déplaisir dont la nature est caractérisée par l'irruption pulsionnelle, source d'angoisse. Cette résistance à l'angoisse est le lot commun du thérapeute et du patient. Le processus analytique commande de résister à la connivence des deux résistances. Le débat dans la cité nécessite que les protagonistes de la cure assument ce débat interne. Sinon c'est la soumission au discours ambiant. L'aliénation ou la fuite signifient l'absence du débat et s'expliquent aussi par la force, le pouvoir d'un tel discours aux confins du moi qui s'appuie défensivement sur une surdité de la souffrance psychique.

«Il est clair que la promotion du moi dans son existence aboutit, conformément à la conception utilitariste de l'homme qui la seconde, à réaliser toujours plus avant l'homme comme individu, c'est-à-dire dans un isolement de l'âme toujours plus parent de sa déréliction originelle.» (Lacan, 1966, 122)

Cependant, pour que cette puissance se maintienne, elle doit se parer de dogmatisme : une affirmation à prétention de vérité mais impensée.

\section{Expression de la rationalité utilitariste}

La rationalité utilitariste conjugue deux intérêts : l'évitement de l'angoisse et la diminution des coûts de la santé mentale. Nous ne nous étonnerons pas de lire, dans le rapport d'un syndicat de soignants, les propos suivants:

«Au Canada, les initiatives récentes en matière de gestion des services de santé semblent converger vers la mise en place de réseaux de services intégrés et l'organisation par programmes-clientèles. Il est à noter que cette organisation a été davantage appliquée au niveau des hôpitaux en médecine physique, que ce soit en Ontario ou au Nouveau-Brunswick, entre autres. Nous n'avons trouvé aucun texte sur l'application des programmes-clientèles en santé mentale ou en psychiatrie. Pourquoi le concept organisationnel de programmes-clientèles a-t-il été appliqué en santé mentale sans 
étude approfondie de ses impacts sur la clientèle desservie? Nous ne pouvons répondre à cette question, nos nombreuses lectures ne nous ayant pas éclairés sur ce sujet. » (SPPASQ, 2005, 3)

La réponse à cette question est superflue, d'une part, parce qu'elle contribuerait à un travail de pensée, ce qui n'est pas requis pour les fins poursuivies et, d'autre part, parce qu'il s'agit ici d'un travail d'organisation, de structuration, pour lequel est requise une pensée opératoire dont les réponses ( $s i$ on y tient) résideraient dans l'explicitation des objectifs. Et comme le mentionne le document, il s'agit de réorganiser les services cliniques en programmes clientèles, c'est-à-dire intégrer les patients dans un programme de consommateurs de soins, programme limité par des impératifs budgétaires.

«S'intéresser aux préoccupations des gestionnaires des services de santé implique rapidement de penser en termes de budgets, de priorités gouvernementales, de modèles organisationnels. L'approche de gestion par programmes-clientèles, au niveau de Louis-H. Lafontaine, dans un contexte de réseaux intégrés de services, au niveau régional, représente le cadre de gestion où nous nous situons en ce moment». (SPPASQ, 2005, 2)

Les prérogatives économiques sont encore plus manifestes dans le rapport du London School of Economics: les rédacteurs proposent d'offrir des thérapies brèves, CBT, (Cognitive Behaviour Therapy) aux patients qui souffrent de dépressions, sous l'égide d'une structure organisationnelle créditée par l'État. Leur argumentaire s'appuie sur les pertes économiques dues à l'absence du monde du travail des patients déprimés qui ne sont donc plus en mesure de payer des impôts ; cette situation a pour conséquence non seulement d'appauvrir l'État, mais, en plus, de représenter un coût pour la collectivité. Les symptômes de la dépression sont pris en charge par les soignants en psychiatrie et ce traitement (prise de médicaments) s'avère coûteux et peu efficace, étant donné que les patients omettent fréquemment de prendre les médicaments.

Ainsi propose-t-on une nouvelle structure: NICE guidelines (National Institute of Clinical Excellence) impliquant des représentants du monde du travail. C'est ainsi que les priorités économiques subordonnent le savoir qui oriente l'intervention clinique. Tout porte à croire que, pour gérer la maladie mentale comme une entreprise rentable, cela exigerait que le politique avec sa législation qui encadre la vie collective, avec le souci d'intégration et d'adaptation par la médiation des institutions, soit assujetti à l'économie.

\section{Fonction du législatif}

On ne s'étonne pas que le politique administre les biens publics à l'intérieur d'un encadrement juridique. Cependant, dans ce cas-ci, il nous semble être en 
présence de la loi qui, dans son application, serait l'équivalent d'un dogmatisme. Je veux ici attirer l'attention sur le fait que la loi, en tant que créature du politique, est non seulement la rationalisation de l'idéologie et de la culture de soins, mais qu'elle est aussi la sauvegarde de ce qui est considéré comme le bien collectif: la priorité du politique. Et, selon l'opinion Jeremy Bentham, le bien politique le plus convoité n'est pas la liberté, mais la sécurité. Le dogme de la sécurité constitueraitil le sous-texte de la législation concernant la maladie mentale? Loi et sécurité deviennent alors les paramètres du bien-être psychique avec le déni de l'angoisse et de la conflictualité. Étant donné que la causalité de ce bien-être est extérieure, gérée par les sujets supposés savoir, nous passons de la psychogenèse à une sociogenèse rationalisée par les sciences comportementales. L'impact de ce passage est d'autant plus coercitif qu'il relève du consensus des institutions du savoir et des soins, en phase avec ce que demande la population lorsqu'elle confie son mal à l'autre, au point de se soumettre à tout discours prescriptif porteur dans l'imaginaire de salut et de guérison.

On peut déceler un aveu derrière cette docilité: je veux devenir autre, désir d'aliénation (alienus : étranger à soi-même), parce que être qui je suis, c'est être souffrant, être patient. Je préfère être client, consommer la médecine et oublier ce malheur de moi-même. Ma souffrance n'a pas à être écoutée, elle a à être irradiée. Voilà la composante principale du dogmatisme: un interdit de savoir sur soi au profit d'un savoir de l'autre, ce gestionnaire de mon bien-être.

«Toute dogmatique manifeste la relation humaine au savoir absolu et fonde la certitude mythologique d'une instance — appelons-la grand $\mathrm{A}$ — d'où procède socialement la parole. Chaque système social est aux prises avec cette affaire. Je dirais, pour résumer, qu'une humanité ne peut être organisée [...] sans l'institution d'un espace où ça sait, où ça sait absolument. » (Legendre, 1983, 32)

\section{Pertinence des thérapies comportementales}

Force est de constater que nous demandons au socius de nous prendre en charge, nous nous présentons en objets de ses bons soins pour notre sécurité. Et en réponse à cette demande, les administrations publiques n'ont d'autre choix que celui de favoriser l'implantation d'approches thérapeutiques qui privilégient l'objectivation plutôt que la subjectivation. Certes, il y a sans doute des résistants à cette tendance lourde mais ils sont marginaux. L'avenir nous dira si leur détermination aura raison des ratés du système.

Mettre mon mal à l'extérieur de moi, l'objectiver évacuant ainsi toute responsabilité. Notons qu'à l'intérieur de cette réflexion, le sens étymologique du terme «responsabilité», respondeo, signifie: «être capable de répondre à partir d'un savoir sur sa subjectivité. » Il semble plus facile d'intervenir sur l'objet, il est plus malléable. Dans ce contexte, les thérapies comportementales sont pertinentes. 
«[...] des sciences comportementales, il ne peut rien sortir, sauf la confirmation de la malléabilité des humains, c'est-à-dire, en définitive, le renforcement, par des moyens modernisés, des techniques classiques du dressage.» (Legendre, 1983, 230)

Nous en déduisons que les deux protagonistes de la cure sont encadrés dans une aliénation réciproque :

«L'aliénation de l'autre est la réalisation d'un désir de mise à mort de la pensée que l'on retrouve présent chez les deux sujets ». (Aulagnier, 1979, 38)

Ainsi assistons-nous à la revendication de la servitude volontaire. Que penser de ce prix à payer pour la poursuite d'un idéal égalitaire qui ne peut se maintenir que par un interdit de savoir?

«J'appelle précisément zone d'ombre cet arrangement, l'ensemble des procédés par lesquels, pour des fins non explicitées [Dans la situation présente on peut présumer que les fins non explicites ont affaire avec des motifs financiers et peut-être aussi l'appétit de pouvoir des corporations professionnelles] fonctionnellement méconnues, l'organisation industrielle parvient non seulement à escamoter la question dogmatique [il faut d'autant plus escamoter la question dogmatique, pour préserver les apparences démocratiques], mais à l'éliminer en tant qu'objet de science. Ceci, de toute évidence, est une notation grave, car du même coup nous soupçonnons que dans nos sociétés hyperscientifiques, un étrange mécanisme est à l'œuvre, maniant en douce et pour ainsi dire à notre insu un interdit de savoir.» (Legendre, 1983, 48)

Or, cet interdit de savoir est la résultante de la complicité existant entre une certaine demande de la population et la nécessité d'encadrer les soins sous l'égide de la loi. Il ne s'agit pas ici de la fonction symbolique de la loi qui est tiers et qui transcende les acteurs : patients, soignants, État. Cette complicité est immanente à la coexistence du maintien de l'ordre et de la dépendance financière et affective : besoin du bénéficiaire de s'aliéner au sujet supposé savoir. À ce titre, le principe de la loi n'est pas une référence mais bien une inférence. Le propre de la loi instaurée par la fonction-tiers est la référence qui permet de passer du monde des sens au monde de l'esprit, passage du primitif (premier, primaire) au civilisé (secondarisé, symbolisé). L'inférence n'en demeure pas moins, si sophistiquée soit-elle, une rationalisation de l'infantile (dépendance, à l'objet imaginaire toutepuissant). 
En clair, «l'absentification» de l'objet-tiers, c'est l'absence de la fonction paternelle sans laquelle le processus de subjectivation est impraticable. La suppression du sujet induit inévitablement la mise de côté de la relation transférocontre-transférentielle. La thérapie prend la forme d'un travail de technicien qui tente de réparer une mécanique défectueuse (un objet). Ne nous y trompons pas, il existe une certaine efficacité dans une telle entreprise. Certains réussissent des modifications de comportements pathologiques. Avec la dextérité de l'horloger, son exactitude et sa précision, le mécanisme se remet en marche. On redevient fonctionnel. L'efficacité de l'approche techniciste fonde la validité de son intervention. L'emprise de l'essence de la technique régissant la science moderne a été critiquée par Heidegger pour qui la technique constitue un oubli de l'être. Le maintien de l'oubli de l'être commande la recherche de l'exactitude et non celle de la vérité.

«De même la non-occultation suivant laquelle la nature se révèle comme un effet complexe et calculable de forces peut sans doute autoriser des constatations exactes; mais, justement en raison de ces succès, elle peut demeurer le danger que le vrai se dérobe au milieu de toute cette exactitude.» (Heidegger, 1992, 35-36)

Le bien-être psychique de l'exactitude, c'est l'éradication du doute. Quelle réponse puis-je espérer à la question sur la vérité de mon désir posée par la médiation de mon symptôme? Une fois de plus, on me répondra: là n'est pas la question. La question est: quoi faire pour supprimer le symptôme?

\section{Appel à la servitude volontaire}

Cette configuration se met en place parce qu'elle est désirée, désir d'être protégé de ce qui pourrait émerger de soi (pulsionnel) qui s'exprime sous le désir de soumission au professionnel tout comme au gestionnaire. Le législatif est inféré de ce désir. Ce qui a pour conséquence que les institutions soignantes sont confinées à contenir la maladie mentale et à répondre au besoin de croire que le salut, la guérison, provient de l'extérieur pour l'obtention de la sécurité. L'impossibilité de remettre en question une telle croyance constitue la nature du dogmatisme dans les politiques de soin.

La raison d'être du dogmatisme est l'ordre social qui tente de maitriser toute potentialité subversive, car la vérité sur le sujet s'avère dangereuse. La résolution de la symptomatique individuelle est donc soumise à la nécessité de l'ordre social. En définitive, ce que l'on tente d'évacuer c'est la tragédie humaine en tant qu'expression de la conflictualité psychique. Science et tragédie, deux irréductibles que nous n'arrivons pas à concilier, à instaurer dans une pensée féconde. Cela nous laisse pratiquement devant une seule issue: le dogmatisme c'est-à-dire une répression de la pensée. 
En définitive, ce qui précède nous ramène à la question proprement analytique : Quels sont les liens entre les effets de l'inconscient et le dogmatisme ? La réponse repose sur le postulat suivant: c'est dans la nature de l'inconscient d'être dogmatique. C'est le retour de l'infantile avec sa prétention imaginaire de toutepuissance, la fusion avec le grand tout collectif en obstruction avec tout processus de subjectivation qui, seul, assure un espace psychique différencié. Cet espace différencié n'est pas donné d'emblée car les capacités de se situer par rapport à autrui, de tolérer et reconnaître nos propres affects et nos propres pulsions comme ceux et celles de l'autre, s'avèrent être un produit aléatoire et il arrive que certains aient besoin d'aide thérapeutique pour y parvenir. Les vicissitudes de la subjectivation et ses empêchements sont indissociables des effets de rencontre avec un environnement souvent éprouvé comme défaillant ou traumatique. (Richard et al., 2006, 1).

Un sujet insuffisamment subjectivé sollicitera plus aisément des modèles, des recettes, des techniques avec l'impératif de fuir toute relation, avec la crainte de répéter les frustrations originaires et cela, en fonction d'un transfert non analysé. Il est privé de la connaissance de lui-même qui pourrait lui donner accès à l'affranchissement des déterminismes qui appauvrissent sa vie. Le problème en cause ici est de percevoir ces déterminismes comme des parasites étrangers et non comme une propriété du sujet. Pour ce point de vue, je me réfère à Green :

«Le sujet commence à comprendre que, quels que soient les facteurs extérieurs qui ont pesé sur son destin (caractère et structure des parents, circonstances historiques générales ou personnelles, environnement plus ou moins défavorable), c'est bien en définitive du sujet qu'il est question, non seulement de la part qu'il a pu prendre à l'organisation de son propre malheur mais du fait que tout ce qu'il a pu vivre comme venant de l'extérieur a bien dû être remodelé à l'intérieur par lui-même, et donc, lui appartient en droit et n'appartient qu'à lui. C'est sans doute là la chose la plus difficile à admettre lorsque certains sujets ont été véritablement victimes de circonstances ou de vicissitudes venant de l'environnement. En fin de compte, après un long travail analytique, le sujet accepte à la fois le fait que les objets de l'environnement n'ont fait que ce qu'ils pouvaient, étant eux-mêmes victimes de leurs propres conflits, et, en plus, comme je l'ai déjà dit ailleurs, que «tout ce qui est en moi fait partie de moi. Tout ce qui a été mis en moi a été réapproprié par moi. Tout ce qui est en moi, en fin de compte, est mien. Mien, c'est-à-dire possession de mon Moi et non pas greffon ou parasite d'organisme qui serait étranger à moi. » (Green, 2002, 127) 


\section{Avènement du sujet}

Le terme même de subjectivation implique qu'il s'agit d'un sujet processuel, se situant au-delà du Moi et de ses mécanismes de défense. C'est la finalité de la démarche analytique conjointement poursuivie avec le processus de deuil. Nous sommes aux antipodes d'une "guérison» causée par l'extérieur du sujet. Aux prises avec sa finitude :

«[...] la vérité du sujet est moins une conquête que l'expérience d'une castration.» (Guyomard, 1998, 56)

Par l'expérience de la castration, l'avènement du sujet implique de renoncer à la démesure du désir et d'assumer, par le deuil de la toute-puissance, l'expérience de sa propre mesure, de ses limites. Or il peut arriver que l'on ne renonce pas et que, confronté à son impuissance, on confie son désir de toute-puissance aux soignants, aux institutions, à l'État. Dans ce cas de figure, les professionnels de la santé et les institutions doivent s'engager dans des processus d'objectivation car du sujet, on ne veut rien savoir. En ce qui a trait à l'intersubjectivité, le technicien ne voudra rien en savoir car n'oublions pas que, dans l'approche analytique, l'intersubjectif ne signifie pas un rapport entre deux individus objectivement identifiables, mais signifie ce qui peut se transmettre entre deux expériences subjectives au sens de deux appareils à penser (appareils psychiques).

L'objectivation telle que le préconisent les sciences «humaines» est un processus qui suppose l'amputation de la dimension pulsionnelle du sujet, ce qui a pour résultat d'aboutir à une entité non-vivante, style mécanique opératoire. Mais il n'en demeure pas moins que ce processus tente d'abolir les affects (l'angoisse, la honte, la culpabilité.) qui peuvent porter préjudice au narcissisme. Le désir narcissique serait le fondement libidinal du désir anti-analytique qui ne peut maintenir ses revendications que par l'abolition de la fonction paternelle (n'y a que la loi de mon désir et tout ce qui viendrait le limiter est jugé répressif et non structurant). Nous sommes en présence d'une revendication, celle de la pleine satisfaction obtenue par la fonction maternelle à amplitude totalitaire. Et si la réalité fait obstruction aux revendications du moi infantile (elle n'y manquera pas), cela déclenchera la destructivité (l'intégrité de mon moi est en danger) dont la décharge prendra les avenues de l'agir ou de la somatisation. Ce qui n'allège pas les coûts sociaux. L'absence de psychisation caractérise ces avenues. Et si la tension n'arrive pas à s'évacuer parce que le retour du refoulé persiste, on devient consommateur d'activités ou de produits autocalmants, bénéficiant d'une satisfaction auto-érotique compensatoire au mal de vivre.

\section{Résistance et dogmatisme}

Au contexte défavorable à l'exercice de la thérapie analytique, on doit ajouter son incompatibilité avec des idéologies comme les systèmes totalitaires, la pensée unique et le capitalisme et ses impératifs financiers. 
«En ce sens, le capitalisme est beaucoup plus qu'un mode de production. C'est une civilisation, c'est une forme de société. II repose sur l'accumulation des hommes utiles, travailleurs et consommateurs à la fois, dotés d'une subjectivité normalisée, c'est-à-dire adaptée à la réquisition dont ils sont les objets. La phrase humaniste selon laquelle "l'homme n'est pas une marchandise comme les autres" sonne tantôt comme une dénégation, tantôt comme un défi selon celui qui la prononce. Mais c'est bien là le "fond de l'affaire".» (Laval, 2007, 324)

Toutefois, il y aura toujours des sujets pour qui la profondeur sera suffisamment érotisée, pour qui la vérité de leur subjectivité sera une recherche essentielle, comme il y aura toujours des sujets «auto-théorisants» (Laplanche). Reconnaissons, par ailleurs, que le clinicien ne peut échapper au dogmatisme ambiant. La qualité de sa réponse, de sa réaction aux pressions sur la conception du thérapeutique, déterminera son avenir. L'une de ses réponses pourrait être de se laisser coloniser par la puissance consensuelle en adoptant un faux-self par rapport à son identité. On se souviendra, à la suite de Winnicott, que la fonction du fauxself est de protéger la fragilité du vrai self, fragilité économique, fragilité identitaire. Assumer son identité analytique n'est pas de tout repos; rencontrer les patients, c'est côtoyer la compulsion de répétition, la tendance à l'agir. Chez certains, nous rencontrons soit des carences élaboratives ou des paroles sans affects, soit une structure masochiste du Moi avec prégnance de la destructivité. Chez d'autres, nous pouvons observer une stagnation dans des régressions ou être confrontés à l'indifférence du patient à l'égard de son univers intérieur. Pourquoi pratiquer ce «métier impossible»? Une réponse adéquate nécessiterait une autre élaboration.

Mais pour persister dans ce «métier», une condition s'impose : être à l'écoute de son inconscient pour explorer la teneur de ses résistances, celles-là mêmes qui participent du désir de ne pas savoir et de ne pas guérir chez les deux protagonistes de la cure.

La résistance n'est pas la seule prérogative de la culture ambiante, mais également l'écueil de tout soignant, y compris le thérapeute d'orientation analytique. Je voudrais attirer l'attention sur l'une de ces résistances qui m'apparaît en collusion avec l'environnement: l'oblitération de la fonction paternelle. On justifie cet effacement en invoquant la fragilité du patient, due à des fixations aux périodes prégénitales qui convoquent un contre-transfert maternel. Le père devient le tiers exclu, c'est-à-dire qu'il n'existe pas dans le désir de la mère. La pertinence de la fonction paternelle, André Green la reconnaît en ces termes :

«J'ai fait l'hypothèse d'une triangulation primitive qui existe même au cœur des échanges dits duels entre la mère et l'enfant. C'est indiquer la place du père, non en tant que personne 
distincte, ce qu'il n'est pas encore au tout début de la vie. Il est néanmoins existant sous la forme de sa présence dans l'esprit de la mère. Cette conception est directement en relation avec la symbolisation.» (Green, 2002, 251)

\begin{abstract}
Absence de symbolisation, absence de travail de deuil, cela conduit à l'immobilisme du travail thérapeutique. Ce qui risque d'avoir pour résultat d'appréhender la mère sous une aura d'autosuffisance et de toute-puissance. Dans ce contexte, la toutepuissance se manifeste dans l'imaginaire comme devant satisfaire tous les désirs infantiles. Cette mère, cet objet nostalgique s'il en est un, est celui-là même auquel l'analyse demande de renoncer. Or, la tentation est grande pour l'analyste de devenir complice du désir infantile. Le thérapeute qui cède à cette idéalisation succombe au désir de prendre la place de l'objet primordial dans sa position aimée, avant l'objet maternel décevant, avant le traumatisme de l'éprouvé de la rage. Contretransférentiellement, nous sommes aux prises avec un moi imaginaire qui entre en complicité avec l'imago maternel du patient. L'emprise de la mère toute-puissante n'est pas sans parenté avec le père de la préhistoire personnelle en tant que
\end{abstract}

«[...] la plus importante identification de l'individu: l'identification au père de la préhistoire personnelle.» (Freud, 1964, 243)

Cette constellation relationnelle agit tel un dogmatisme. Ce qui n'est pas sans influence sur les impasses de la relation duelle dans la cure. Cette relation circulaire, sous la pression du désir de réparation narcissique, devient pour le couple thérapeutique un enfermement et le processus du travail psychique risque de stagner, ce qui peut avoir des effets dissuasifs chez les thérapeutes tout en alimentant les critiques à l'endroit de l'approche analytique. Je mentionnerai que la loi induite par une mère sans père est une loi «inférentielle » ce qui est syntone à notre époque. Si c'est un effort d'adaptation de notre part pour ne pas être exclus du champ social, le résultat obtenu est que nous nous excluons de nous-mêmes.

Ce n'est pas la fermeture, l'hostilité en soi de l'approche analytique qui représente la plus grande difficulté, c'est l'introjection de cette fin de non-recevoir qui s'introduit dans l'instance surmoïque. Je pense à l'anecdote suivante: un collègue analyste conseille à une jeune psychologue en quête d'emploi «surtout ne leur dites pas que vous êtes d'orientation analytique». Subrepticement, s'infiltre l'idée que l'identité analytique est abandonnée par la communauté soignante. À partir de là, il n'y a qu'un pas pour devenir travailleur clandestin. C'est la réflexion que m'inspiraient les propos de ce thérapeute: «Je ne dis pas que je suis psychologue d'orientation analytique parce qu'on ne m'enverra pas de patient. » Pourtant ce professionnel vient en supervision avec une psychanalyste. Le désir d'analyse, de compréhension analytique, est-il devenu une atteinte aux exigences objectives du conformisme social? Ce qui est en cause, c'est l'estime publique, la reconnais- 
sance officielle marquée par des bénéfices pécuniaires, plus nous en sommes dépendants, plus nous sommes susceptibles de compromissions. En fait, notre dépendance précède les contraintes sociales. On s'autorise en cabinet privé, on s'interdit sur la place publique, à moins que la place publique se limite à la communauté analytique Même à l'intérieur des sociétés analytiques, certains se refuseront la prise de parole ou même au travail de l'écrire, de peur des jugements dépréciatifs de la part de leurs pairs. Leur conception de l'analyse s'enferme dans le secret.

Et si le public représente transférentiellement une figure parentale, l'interdit du désir analytique est renforcé. Interdit parental qui est représenté par refus de soi en tant que sujet, pour laisser la place à l'objet : celui du désir de l'Autre. En conséquence, la parole analytique est muselée. Ici, contrairement à ce qu'il est habituel de dire, ce n'est plus l'offre qui précède la demande, c'est la demande (l'exigence) qui précède l'offre. Éventuellement l'offre est diluée, «adaptée» sur le plan clinique. Sur le plan théorique (élaboration, modification de cadre), on tente de justifier l'offre en fonction de la demande. On constate également un autre phénomène, celui de l'attraction auprès d'adeptes séduits par les aspects intellectuels de la psychanalyse, mais qui ne s'y engagent pas véritablement. Je qualifierais ces adeptes de dilettantes de la psychanalyse. Ce qui est questionnable, ce n'est pas tant leur intérêt pour l'analyse, c'est l'absence d'analyse de leur résistance.

L'analyse des résistances me paraît le parcours obligé pour maintenir une identité analytique. À mon sens, elle ouvre sur une compréhension de notre époque, car les bruits du monde sont l'écho de nos bruits intimes. En fin de parcours d'une réflexion sur les déterminismes qui conditionnent l'avenir du clinicien, je suis portée à penser que, pour assurer son avenir, il faut tenir compte, dans nos réflexions et nos interventions, des déterminismes suivants : résistance, dogmatisme en lien dialectique avec l'identité du clinicien d'approche analytique. Pour y arriver, il m'apparaît nécessaire d'enrichir la connaissance de la vie psychique par la prise en compte des données du monde, sans omettre d'assumer l'héritage subversif de la psychanalyse. Et pour exister dans la cité, on ne peut échapper à l'obligation de rendre compte du travail de la pensée, de l'écoute de l'inconscient par la prise de parole dans le social.

claudette lafond

10135, rue saint-denis montréal, québec

h31 2h9

\section{Bibliographie}

Aulagnier, P. , 1979, Les destins du plaisir, Paris, P.U.F.

Chabert, C., 2002, Les hommes ne veulent pas guérir, in La résistance, Actes des entretiens de psychanalyse de l'A.P.F. 


\section{8}

Filigrane, automne 2008

Freud, S., 1936, Lettre du 22 mars, citée par Ernest Jones, in La vie et l'œuvre de Sigmund Freud, tome 3, Paris, Presses Universitaires de France, 1969.

Freud, S., 1925, Ma vie et la psychanalyse, Paris, Gallimard, 1950.

Freud, S., 1923, Le moi et le Ça, in Essais de psychanalyse, Petite Bibliothèque Payot, 1985.

Green, A., 2002, Idées directrices pour une psychanalyse contemporaine, Paris, P.U.F.

Granoff, W., 2001, Lacan, Ferenczi et Freud, Paris, Gallimard.

Guyomard, P., 1998, Le désir d'éthique, Paris, Aubier.

Heidegger, M., 1992, Essais et conférences, Paris, Gallimard.

Laval, C., 2007, L’homme économique. Essai sur les racines du libéralisme, Paris, Gallimard.

Lacan, J., 1948, L'agressivité en psychanalyse Paris, in Écrits, Paris, Le Seuil.

Legendre, P., 1983, Leçons II. L'empire de la vérité (Introduction aux espaces dogmatiques industriels), Paris, Fayard. Richard, F., Wainrib, S., 2006, La subjectivation, Paris, Dunod

Varela, F., Thompson, E., Rosch, E., 1991, trad. française, (1993), L'inscription corporelle de l'esprit, Paris, Seuil, 